\title{
Suspensions of prolate spheroids in Stokes flow. Part 3. Hydrodynamic transport properties of crystalline dispersions
}

\author{
By IVAN L. CLAEYS $\dagger$ AND JOHN F. BRADY \\ Division of Chemistry and Chemical Engineering, California Institute of Technology, Pasadena, \\ CA 91125, USA
}

(Received 14 August 1991 and in revised form 18 October 1992)

The short-time limit of the hydrodynamic transport properties is calculated for crystalline dispersions of parallel prolate spheroids using a moment expansion technique similar in concept to the simulation method known as Stokesian dynamics. The concentration dependence of the sedimentation rate, the hindered diffusivity and the rheological behaviour of face-centred lattices are examined for concentrations up to regular close packing ( $74 \%$ by volume). The influence of the detailed microstructure of the dispersion is also investigated by considering different arrangements of parallel ellipsoids. Useful reference configurations are proposed as standard geometries for regular arrays of prolate spheroids.

\section{Introduction}

Hard-rod dispersions undergo a phase transition to a liquid crystalline state at high volume fractions (Onsager 1949; Frenkel 1987). This sudden change in the equilibrium microstructure, caused solely by excluded volume effects, dramatically alters the macroscopic characteristics of the material. Most notably, the anisotropy of the microscopic configuration imparts a directionality to most observable properties, including all hydrodynamic transport coefficients. The growing impact of liquid crystals on industrial and household technology drives many current research efforts in this area. Microstructural theories, which explain macroscopic phenomena in terms of the underlying physics at the mesoscale, have, in conjunction with Monte-Carlo and molecular dynamics simulations, already greatly expanded our understanding of these complex systems under equilibrium conditions (Allen, Frenkel \& Talbot 1989; Talbot et al. 1990). Yet most of these works either consider fluids of hard convex bodies (Frenkel \& Mulder 1985; Talbot et al. 1990), or neglect hydrodynamic interactions between the rods while recognizing that this significantly restricts the scope and validity of the analysis (Bitsanis, Davis \& Tirrell 1988, 1990). The simulation method which we introduced in two companion papers (Claeys \& Brady 1993a,b), on the other hand, is ideally suited to investigate the rheological properties of liquid crystalline domains.

At high concentrations, a fluid of hard prolate spheroids spontaneously assembles into a structure devoid of translational order, but possessing a preferred direction of alignment (Onsager 1949; Frenkel, Mulder \& McTague 1984). Such textures are called nematics. At higher densities still, it solidifies into a crystal resembling a face-centred cubic cell of spheres stretched along the (111)-axis (see below). In this work, we shall consider dispersions of spheroids characterized by perfect orientational and translational order, whose microstructure near the maximum packing is similar to the

$\dagger$ Present address: Solvay Research \& Technology, rue de Ransbeek 310, 1120 Brussels, Belgium. 
equilibrium crystal of ellipsoids. At lower densities, these systems can be viewed as models for liquid crystalline domains. This picture of nematics is admittedly overly simplified considering the complex molecular arrangement of true liquid crystals. In particular, the extent of translational order is very limited in real systems, and the particles' alignment is far from perfect. Both factors significantly affect the macroscopic properties of the dispersion, such as its extensional viscosity (Claeys \& Brady 1993b). More representative equilibrium microstructures can be generated by Monte-Carlo techniques or by molecular dynamics. Model constructions such as the one proposed here however, are valuable analytical tools as they often allow straightforward interpretations of complex physical phenomena. These idealized structures also serve as useful reference configurations, comparable to simple cubic lattices for spheres (Appendix A). We calculate the short-time limit of the hydrodynamic transport properties of these dispersions according to the methods presented earlier (Claeys \& Brady $1993 a, b)$. As before, the particles are non-Brownian and monodisperse.

The following section defines the efc-geometry and elaborates on the advantages of this standard structure. We report on its properties in the next three sections, beginning with the sedimentation rate and ending with the rheological characteristics. Hindered diffusion in efc-crystals is also briefly discussed. The last section examines the influence of the detailed topology of the dispersions on their rheological properties. The relations between the stress in the suspension and the imposed rate of strain are compared for five different lattices of parallel spheroids and their differences are explained in terms of the geometric features of each arrangement.

\section{Definition of the efc-lattice}

The highest packing fraction attainable for prolate spheroids on a simple cubic cell is $\pi /\left(6 r_{p}^{2}\right)$, and hence is about $0.5 \%$ for rods of aspect ratio 10 . The aspect ratio, $r_{p}=a / b$, is defined as the ratio of the lengths of the major and minor semi-axes, respectively. It is possible, however, to achieve a density equal to the maximum for spheres, $\pi /(3 \sqrt{ } 2)$, by stretching a face-centred cubic cell of spheres in any direction by a factor equal to the aspect ratio of the spheroids. This transformation maps a sphere onto an ellipsoid without altering the volume fraction of the crystal. To preserve as many symmetry elements in the lattice as possible, we chose the direction of stretch to be perpendicular to the hexagonally packed stacks, i.e. along the (111)-diagonal of the original cubic cell (figure 1). This guarantees the simplest possible form for the tensorial properties of the crystal. We shall refer to the direction of stretch, which coincides with the orientation of the spheroids, as the $z$-axis. The system thus possesses 6-fold symmetry about $z$. Based on the invariance of the microstructure under rotations by $\frac{1}{3} \pi$ in the $(x, y)$-plane, and under reflections about the origin, one can show for instance that the fourth-rank tensor which relates the stress to the rate of strain only has three independent components. (Using only the tracelessness of $\boldsymbol{S}$ and $\boldsymbol{E}$ and the symmetry of $\boldsymbol{R}_{S E}$, one cannot reduce the number of coefficients below 15. For comparison, four coefficients are needed if the lattice is stretched in the (001) direction, and only two for face-centred cubic cells (Nunan \& Keller 1984).) In addition, a distortion along (111) generates a structure which resembles the configuration into which fluids of hard ellipsoids solidify at high densities. It is thus physically realizable in this sense.

In order to study dispersions at concentrations different from the maximum, an algorithm must be devised to expand the lattice. The most simple-minded approach, an isotropic dilatation, soon results in very uninteresting structures consisting of hexagonally packed sheets of spheroids separated by large gaps filled with fluid, not at 

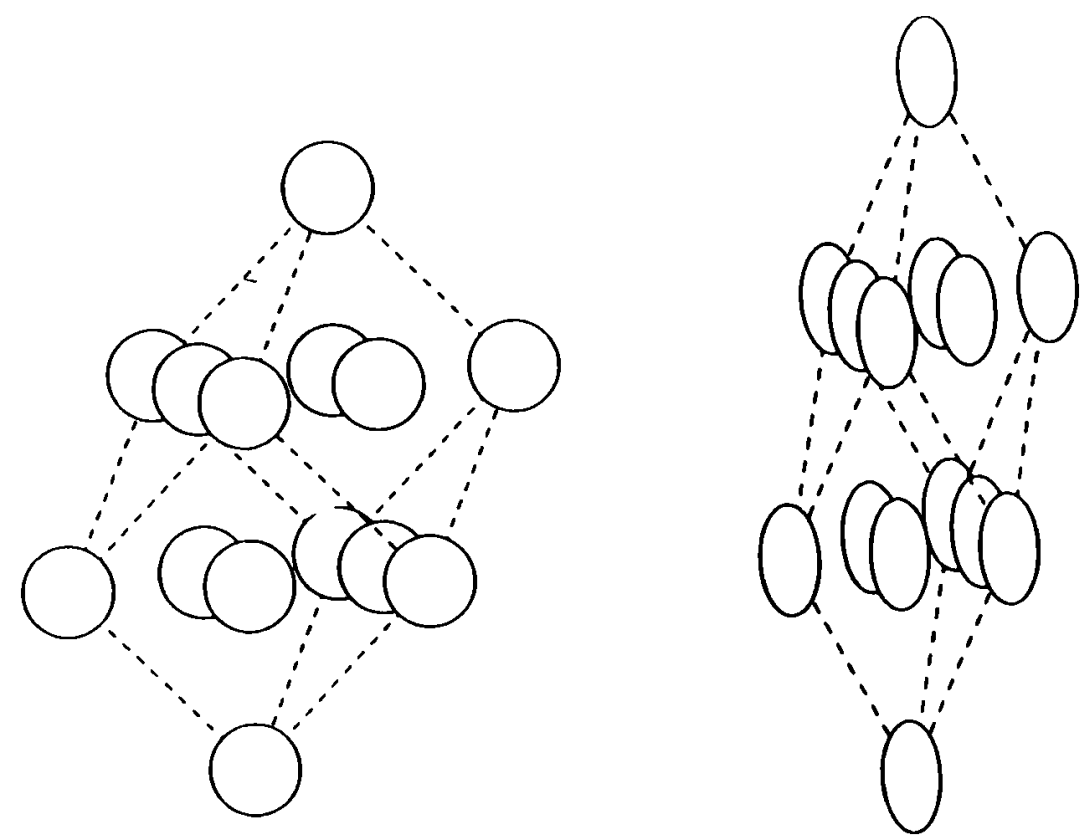

Figure 1. Expansion of a face-centred cubic lattice of spheres into an efc-lattice of spheroids. The direction of stretch is perpendicular to the hexagonally packed planes.

all reminiscent of the liquid crystalline domains that we wish to model. This is a consequence, of course, of the marked shape anisotropy of the unit cell at the highest volume fraction. In order to preserve a 'space-filling' configuration even at low concentrations, we decided to expand the lattice in such a way that the surface-tosurface separation between nearest neighbours is the same in all directions (Appendix A). Although this definition is somewhat arbitrary, it offers an unambiguous way of constructing an 'isotropically compact' structure with full three-dimensional character. This has the added benefit that the geometry reduces to a face-centred cubic geometry at infinite dilution, independently of the aspect ratio of the particles. We shall call this type of crystalline geometry 'expanded face centred', or efc for short.

The entire crystal can be built using only one spheroid per unit cell. This has obvious computational advantages, since the time savings associated with using the minimal number of particles far outweighs the cost of having a denser lattice over which to perform the Ewald summation (Claeys \& Brady 1993 b). Unfortunately, this also impoverishes the information content of the simulations; indeed, because the forcing is periodic, the problem formulations for the self-diffusivity $D$, the hindered diffusion coefficient $\boldsymbol{H}$ and the sedimentation rate $\boldsymbol{V}$ become identical, and no meaningful results can be obtained for $\boldsymbol{D}$ nor $\boldsymbol{H}$ unless more particles are included in the unit cell. Nevertheless, because of its efficiency, we used the most elementary module, containing a single spheroid, for most of the results reported here. Note that the basis vectors of the unit cell are non-orthogonal and of different lengths, but the resulting geometric complications are relatively straightforward to overcome.

\section{Sedimentation rates}

The sedimentation rates for an efc-lattice of spheroids of aspect ratio 6 , normalized by their value at infinite dilution, are displayed in figure 2 , alongside their rotational counterparts (i.e. the rotation rates due to uniformly applied torques). From symmetry 


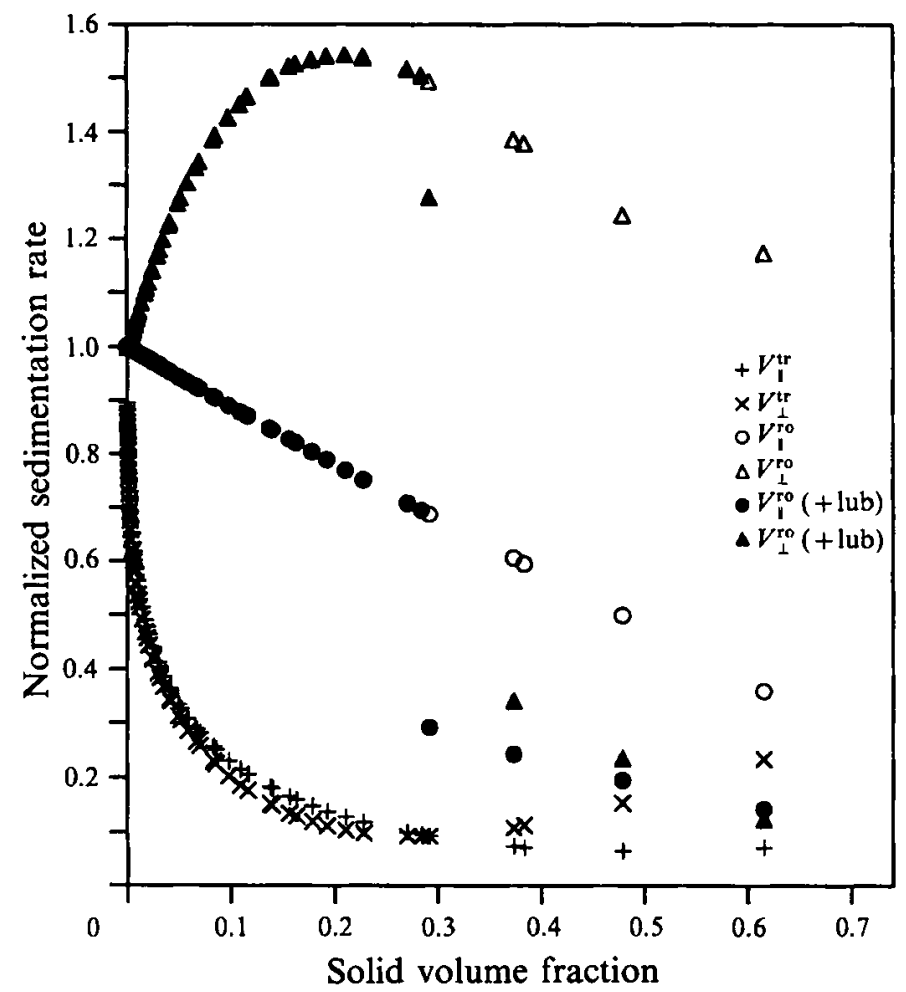

FIGURE 2. Sedimentation rates of efc-lattices of spheroids of aspect ratio 6 . The sedimentation rates are normalized by their value at infinite dilution.

considerations, all particles fall at the same speed, and there is no coupling between translation and rotation. Motions along the direction of alignment are also decoupled from motions perpendicular to it. Furthermore, as pointed out earlier, the crystal possesses a 6-fold axis of symmetry and thus is isotropic in the $(x, y)$-plane. Therefore, four components suffice to characterize the $6 \times 6$ sedimentation tensor (or collective diffusion tensor): $V_{\|}^{\text {tr }}, V_{\perp}^{\text {rr }}, V_{\|}^{\text {ro }}$, and $V_{\perp}^{\text {ro }}$. The rotational 'sedimentation rate' $V_{\|}^{\text {ro }}$ represents the angular velocity of the particles resulting from a uniformly applied torque parallel to their axes; $V_{\perp}^{\text {ro }}$ is defined in a similar fashion.) Perhaps the most striking feature of figure 2 is the peculiar concentration dependence of the collective rotational mobility perpendicular to the rod axis. A very shallow minimum at an extremely dilute volume fraction ( $V_{\perp}^{\mathrm{ro}}$ reaches 0.99933 at $\phi=0.00035$ ) is followed by a pronounced maximum exceeding 1 at about $21 \%$ solids by volume. The initial drop is due to the increased effective viscosity of the suspension. The subsequent rise can be understood by reference to figure 3 , where we sketch how viscous interactions between the rods reinforce the particles' rotation, in a manner similar to the drag reduction experienced by two spheres when they fall as a doublet rather than as two isolated balls. At higher concentrations, however, the hexagonally packed stacks of spheroids become interpenetrating, and actively counteract each other, causing the collective rotational diffusivity $V_{\perp}^{\text {ro }}$ to drop again. A schematic drawing similar to figure 3 would show that hydrodynamic interactions always act to retard the motion in the case of $V_{\|}^{\text {ro }}$, which therefore decreases monotonically with increasing volume fraction. The translational sedimentation rates $V^{\mathrm{tr}}$ also fall off with concentration, as expected intuitively, because the strong backflow overwhelms the analogous cooperative effect of hydrodynamic interactions. The slight upward bend in $V^{\mathrm{tr}}$ at high packing densities is associated with 

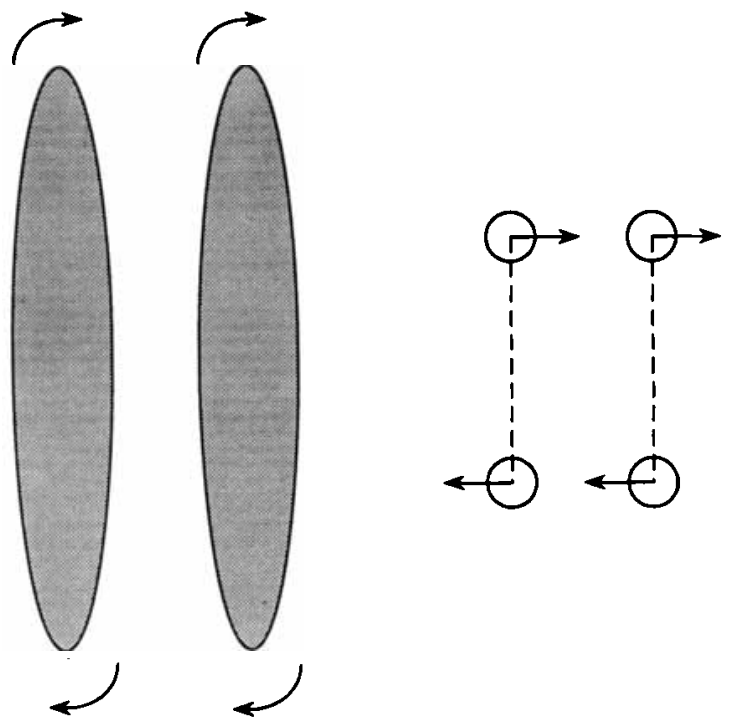

FIGURE 3. Illustration of the mechanism leading to enhanced collective rotational diffusivities $V_{\perp}^{\text {ro }}$ in efc-crystals of ellipsoids.

the neglect of multipoles higher than the torque and the stresslet, and should be disregarded. (Similar aphysical behaviour has been noted in simulations of concentrated samples of spheres if the mean field quadrupole was not included in the formulation, as is the case here.)

Symmetry arguments demonstrate why lubrication stresses affect $V^{\text {ro }}$, but not $V^{\text {tr }}$. This is true for every microstructure which can be constructed using a single particle per unit cell. The abruptness of the drop at about $28.5 \%$ is artificial, and merely a consequence of our lack of knowledge concerning the $O\left(\epsilon^{0}\right)$ correction to the lubrication formulae of Claeys \& Brady (1989). This $O(1)$ constant provides the smooth transition from the 'far-field' behaviour to the 'near-field', singular resistance behaviour as the surface separation $\epsilon$ between two particles decreases. Without it, lubrication interactions must be allowed to 'kick in' at a certain threshold separation $\epsilon_{\max }$, set at 1 in these simulations. (This distance is non-dimensionalized by the harmonic mean of the radii of curvature at the points of closest approach.) The effect is quite dramatic for crystals because lubrication stresses set in at the same concentration for all particles. No discontinuity is noticeable for more random configurations, since the contribution of lubrication stresses to macroscopic properties is smeared out by averaging over the particles in that case (cf. the results on equilibrium hard-spheroid dispersions (Claeys \& Brady $1993 b$ )). Moreover, the threshold $\epsilon_{\max }$, which is somewhat arbitrary, was set at an unusually high value here for illustrative purposes. We typically chose $\epsilon_{\max }=0.08$, which is more consistent with the assumption underlying lubrication theory that $\epsilon \ll 1$. In the remainder of this article, we shall actually suppress lubrication effects altogether $\left(\epsilon_{\max }=0\right)$.

For a suspension of spheres of radius $a$ arranged on a simple cubic lattice of volume fraction $\phi$, Hasimoto (1959) calculated that

$$
V^{\mathrm{tr}}=1-1.74 \phi^{\frac{1}{3}},
$$

where the sedimentation velocity has been scaled by $|F| / 6 \pi a \mu$, with $\mu$ the dynamic viscosity of the suspending fluid. The collective translational mobilities $V^{\text {tr }}$ of spheroids on an efc-lattice do not, however, decrease quite linearly with the cube root of the 


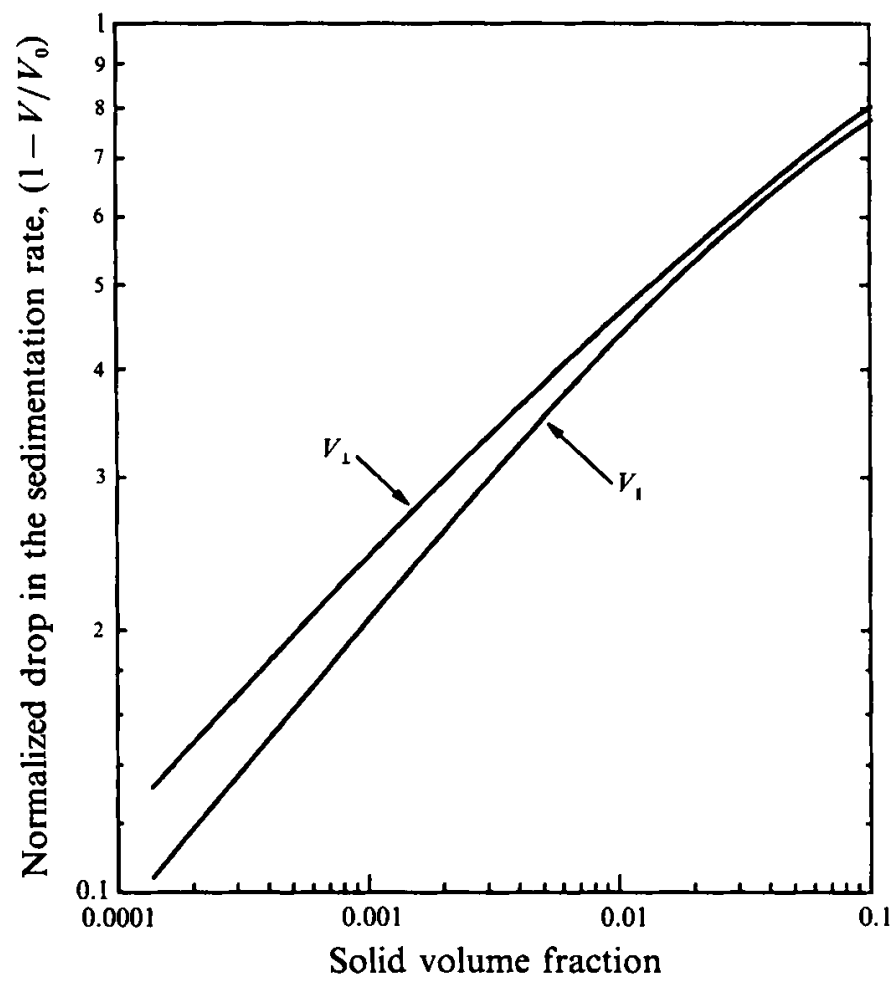

FIGURE 4. Asymptotic dependence of the sedimentation rate of efc-crystals on the volume fraction of particles $\left(r_{p}=6\right)$. For reference, note that the diagonal of the graph has a slope of $\frac{1}{3}$.

concentration, although this dependence is approached asymptotically (figure 4). This deviation is explained by the fact that the lattices do not remain perfectly self-similar when they are compressed, because they are constructed so that the superficial separation between the spheroids is uniform in three non-planar directions (vide supra).

The sedimentation rate $V_{\perp}^{\mathrm{tr}}$ drops faster with concentration than $V_{\|}^{\mathrm{tr}}$ for all aspect ratios examined. The decline steepens slightly as the eccentricity of the spheroids increases. The maximum in $V_{\perp}^{\text {ro }}$ also become higher, reaching more than 10 for $r_{p}=24$ at $\phi \approx 0.21$.

\section{Hindered diffusivities}

As explained earlier, the evaluation of hindered diffusion coefficients requires the use of more than one particle per unit cell. Figure 5 shows the hindered diffusivities for translation and for rotation perpendicular to the rod axis in an efc-lattice of spheroids of aspect ratio 6 obtained using 1, 4, 8,16 and 32 ellipsoids per unit cell. In the last case, one particle is subject to a force (or torque, respectively), and the remaining 31 are held still. The set of data with $N=1$ reproduces the sedimentation rates. For both translational and rotary motion, the number dependence is rather mild, and the results for 32 particles are not much different from the ones for $N=16$. The rotational mobility $H_{1}^{\mathrm{To}}<V_{1}^{\mathrm{ro}}$, because the cooperative hydrodynamic interactions giving rise to the maximum in the $\phi$-dependence of $V_{\perp}^{\text {ro }}$ are screened by the intervening stationary particles. For the translational motion, $H_{\perp}^{\mathrm{tr}}>V_{\perp}^{\mathrm{tr}}$ because the backflow of fluid, driven by the pressure gradient balancing the forces acting on the particles, weakens (from a macroscopic momentum balance), becoming zero as $N \rightarrow \infty$. 


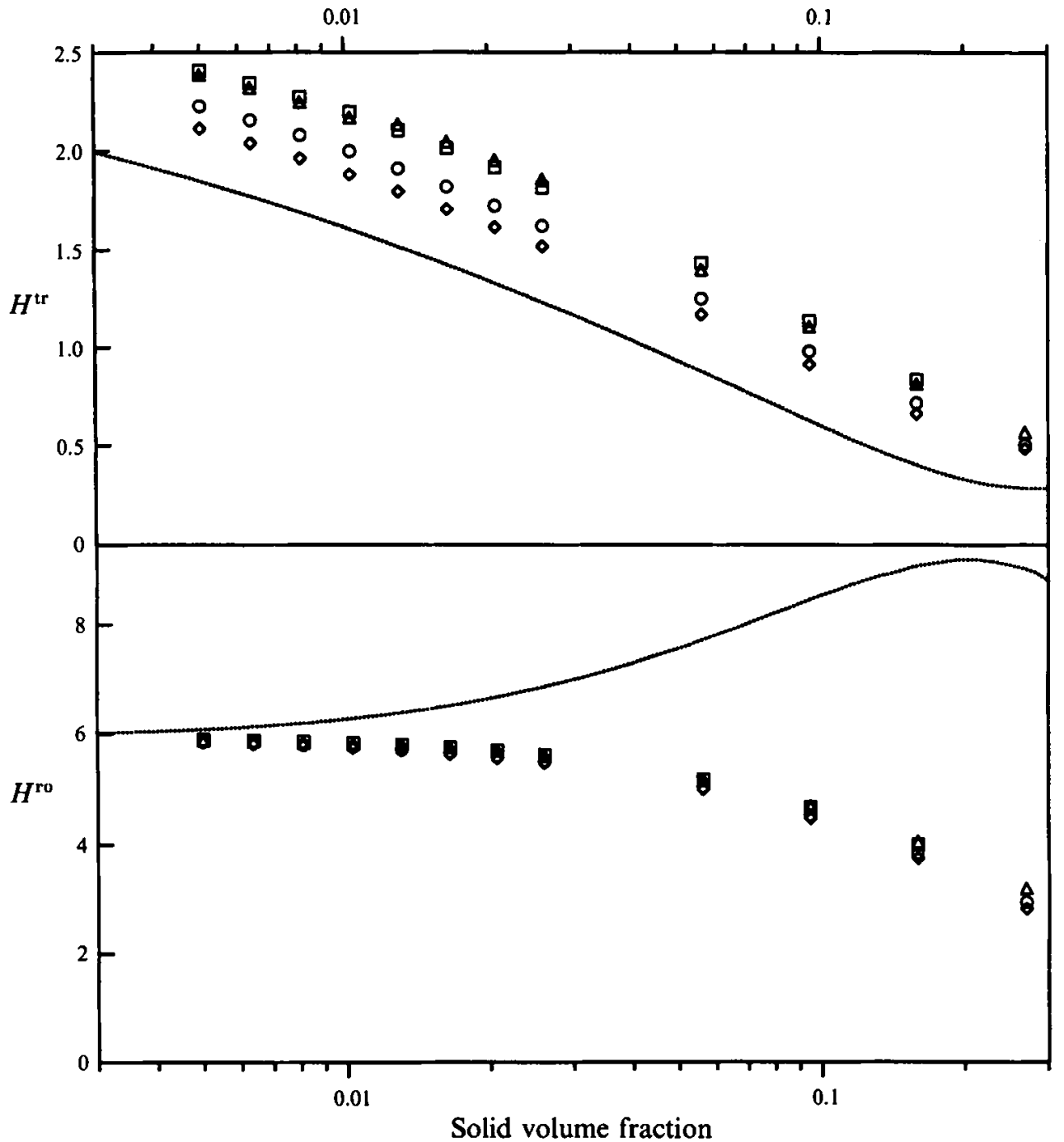

FIGURE 5. Hindered diffusivities $H_{\perp} v s$. volume fraction for efc-lattices of prolate spheroids of aspect ratio 6. The diffusion coefficients are normalized by $k T / 8 \pi a^{n} \mu$, with $n=1$ for $H^{\mathrm{r}}$ and $n=3$ for $H^{\mathrm{ro}}$. The lines represent data for one particle per unit cell and correspond to the sedimentation rate and its rotational analog. The unit cell for $N=4(\diamond)$ was constructed by including the images closest to the origin in the hexagonally packed plane. These were then replicated one basis vector away to build a unit cell containing eight particles $(O)$. This procedure was repeated to obtain $N=16(\triangle)$ and $32(\square)$.

\section{Relation between the stress and the rate of strain}

The hydrodynamic stress in a suspension of force-free particles subject to a flow with bulk average rate of strain $\boldsymbol{E}_{\infty}$ is given by (Batchelor (1970)

$$
\Sigma=-p_{\mathrm{etf}} \delta+2 \mu \boldsymbol{E}_{\infty}-n_{\phi}\langle\boldsymbol{S}\rangle .
$$

The isotropic pressure term is irrelevant in incompressible media, and the second term represents the Newtonian fluid phase response. The last term, proportional to the number density $n_{\phi}$ of particles in the dispersion, relates the hydrodynamic stress to the mean stresslet $\langle\boldsymbol{S}\rangle$ exerted by the solid bodies. This stress moment can be calculated 

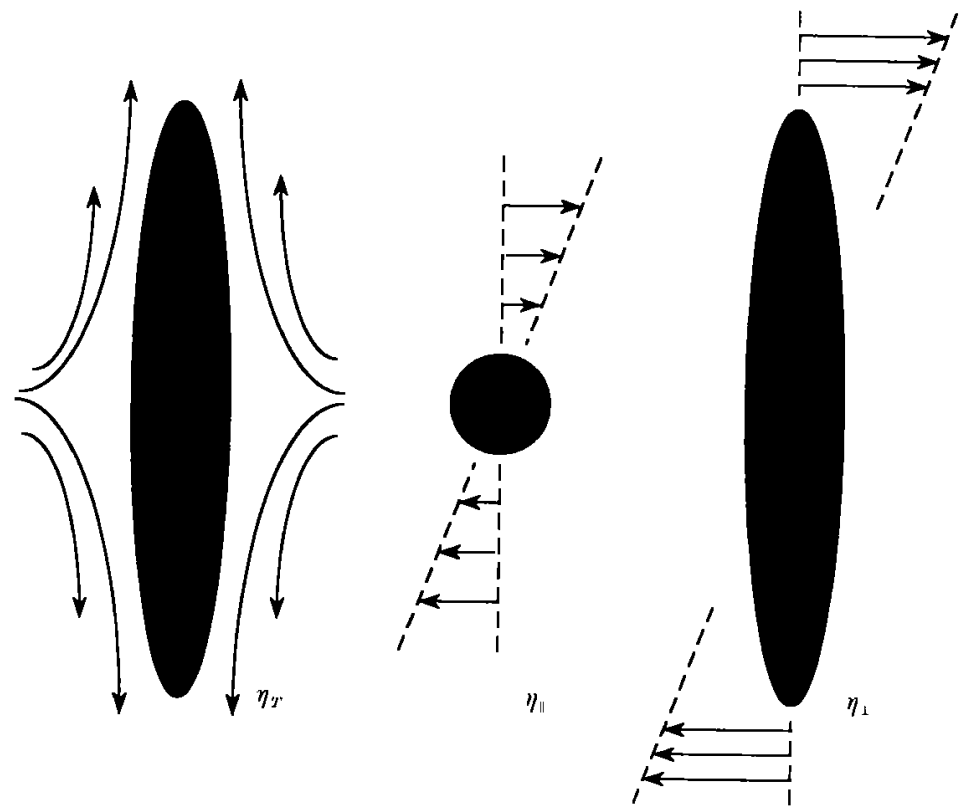

Figure 6. Shear fields associated with the three coefficients characterizing the relation $\boldsymbol{R}_{\Sigma E}$ between the stress and the rate of strain for efc-lattices (equation (5.3)).

by contracting the appropriate elements of the grand resistance tensor (Brady \& Bossis 1988):

$$
\langle\boldsymbol{S}\rangle=\left\langle\boldsymbol{R}_{S \Psi l} \cdot \boldsymbol{R}_{\# \sharp}^{-1} \cdot \boldsymbol{R}_{\mathscr{F} E}-\boldsymbol{R}_{S E}\right\rangle: \boldsymbol{E}_{x_{x}} .
$$

(This notation for the resistance tensors is standard and fairly transparent. $\boldsymbol{R}_{S^{*}}$ for instance relates the stresslets $\boldsymbol{S}$ on the particles to their motion $\mathcal{U}$, which includes both translational and angular velocities. Similarly, $\mathscr{F}$ groups the forces as well as the torques exerted by the particles (Claeys \& Brady 1993b).)

By applying the transformations for which the efc-lattice is invariant (a rotation by $\frac{1}{3} \pi$ about the $z$-axis, for instance), exploiting the tracelessness and symmetry of the stresslet $\boldsymbol{S}$ and of the rate-of-strain tensor $\boldsymbol{E}$, and making optimal use of the implications of Lorentz' reciprocal theorem (Hinch 1972), it is easy to demonstrate that

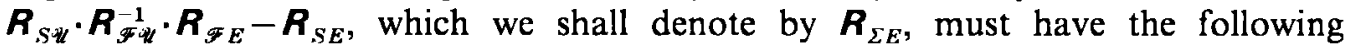
structure:

$$
\begin{aligned}
\boldsymbol{R}_{\Sigma E}= & 8 \pi a^{3} \mu\left\{3 \eta_{T^{T}}\left(d d-\frac{1}{3} \delta\right)\left(d d-\frac{1}{3} \delta\right)\right. \\
& +\eta_{T^{\top}}\left(d \delta d+{ }^{\mathrm{T}} d \delta d+d \delta d^{\mathrm{T}}+{ }^{\mathrm{T}} d \delta d^{\mathrm{T}}-4 d d d d\right) \\
& \left.+\eta_{\|}\left(\boldsymbol{\delta} \delta+\hat{\delta} \delta-\delta \delta+d d \delta+\delta d d+d d d d-d \delta d-{ }^{\mathrm{T}} d \delta d-d \delta d^{\mathrm{T}}-{ }^{\mathrm{T}} d \delta d^{\mathrm{T}}\right)\right\} .
\end{aligned}
$$

The particle half-length $a$ and the viscosity $\mu$ of the suspending fluid are used to nondimensionalize $\eta$. The unit vector $d$ gives the orientation of the rods, and lies along the $z$-axis. The non-standard notations $\hat{\delta} \hat{\delta}$ and $\widetilde{\delta} \overline{\text { mean }}$

$$
(\check{\delta})_{i j k l}=\delta_{i l} \delta_{j k} \text { and }(\hat{\delta} \hat{\delta})_{i j k l}=\delta_{i k} \delta_{j l} \text {. }
$$

To within at least five significant digits, the tensor $\boldsymbol{R}_{\Sigma E}$ calculated for an efc-lattice using Stokesian dynamics conformed to (5.3). For isotropic suspensions, the three coefficients $\eta_{T}, \eta_{\perp}$ and $\eta_{\|}$are equal to the scaled single-particle contribution $\eta$ to the effective viscosity [i.e. $\boldsymbol{R}_{\Sigma E}=8 \pi a^{3} \mu \eta\left(\delta \delta+\delta \delta-\frac{2}{3} \delta \delta\right)$ so that

$$
\left.\mu_{\text {eff }}=\mu\left(1+8 \pi a^{3} n_{\phi} \eta\right)=\mu\left(1+6 r_{p}^{2} \phi \eta\right)\right] .
$$




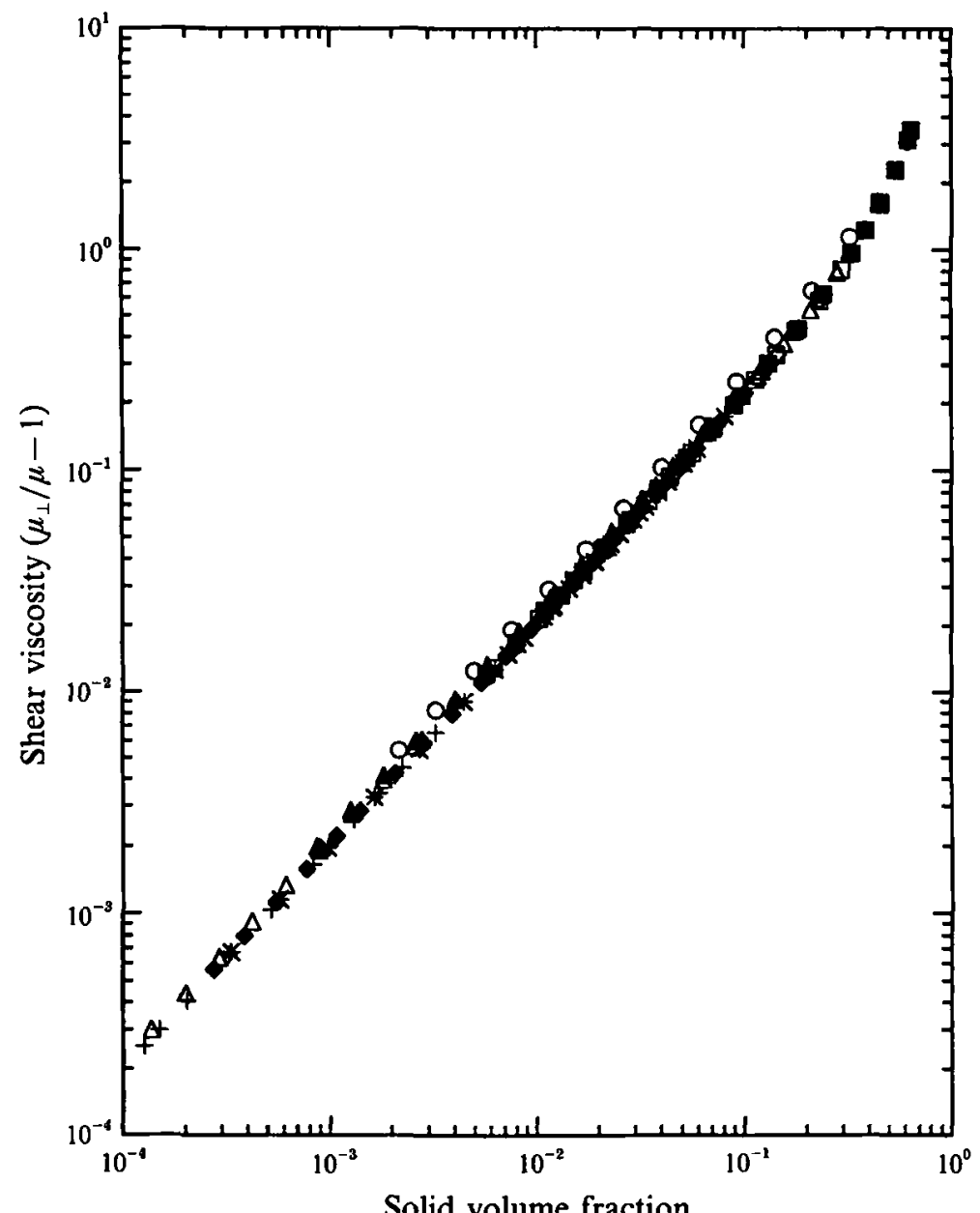

FIGURE 7. Particles' contribution to the apparent shear viscosity $\mu_{\perp}=\mu\left(1+6 r_{p}^{2} \phi \eta_{\perp}\right)$ of efc-lattices of spheroids. The symbols correspond to various aspect ratios and are explained in the legend of figure 8 . Lubrication interactions have been suppressed. The data for $\mu_{\|}$would be indistinguishable on this plot.

For dispersions which possess cylindrical symmetry about $d$ (such as efc-lattices of spheroids), $\eta_{T}$ gives the resistance to uniaxial extension in the direction of $d, \eta_{\perp}$ indicates the stress in response to simple shearing flows with a velocity gradient parallel to $d$, and $\eta_{\|}$corresponds to the apparent viscosity during simple shear (or hyperbolic straining) in a plane perpendicular to $d$ (figure 6).

At infinite dilution, (5.3) reduces to the expression for the stresslet exerted by an isolated freely mobile spheroid in a purely extensional flow. Using the symbols of Chwang \& Wu (1975) (see also Kim 1986), we can derive the limiting forms of $\eta_{T}, \eta_{\perp}$ and $\eta_{\sharp}$ for very slender rods as

$$
\begin{gathered}
\eta_{T} \stackrel{n_{\phi} \rightarrow 0}{\rightarrow}-\frac{2}{9} e^{3} \alpha_{5} \stackrel{e \rightarrow 1}{\rightarrow}\left(18 \log \left(2 r_{p}\right)-27\right)^{-1}+O\left(r_{p}^{-2}\right), \\
\eta_{\perp} \stackrel{n_{\phi}>0}{\rightarrow} \frac{1}{3} e^{3}\left(\gamma^{*} \frac{e^{2}}{2-e^{2}}-\alpha^{*}\right) \stackrel{e \rightarrow 1}{\rightarrow} r_{p}^{-2}+O\left(r_{p}^{-4}\right), \\
\stackrel{n_{\phi} \rightarrow 0}{\rightarrow}-\frac{2}{3} e^{3} \alpha_{4} \stackrel{e \rightarrow 1}{\rightarrow} \frac{1}{3} r_{p}^{-2}+O\left(r_{p}^{-4}\right),
\end{gathered}
$$




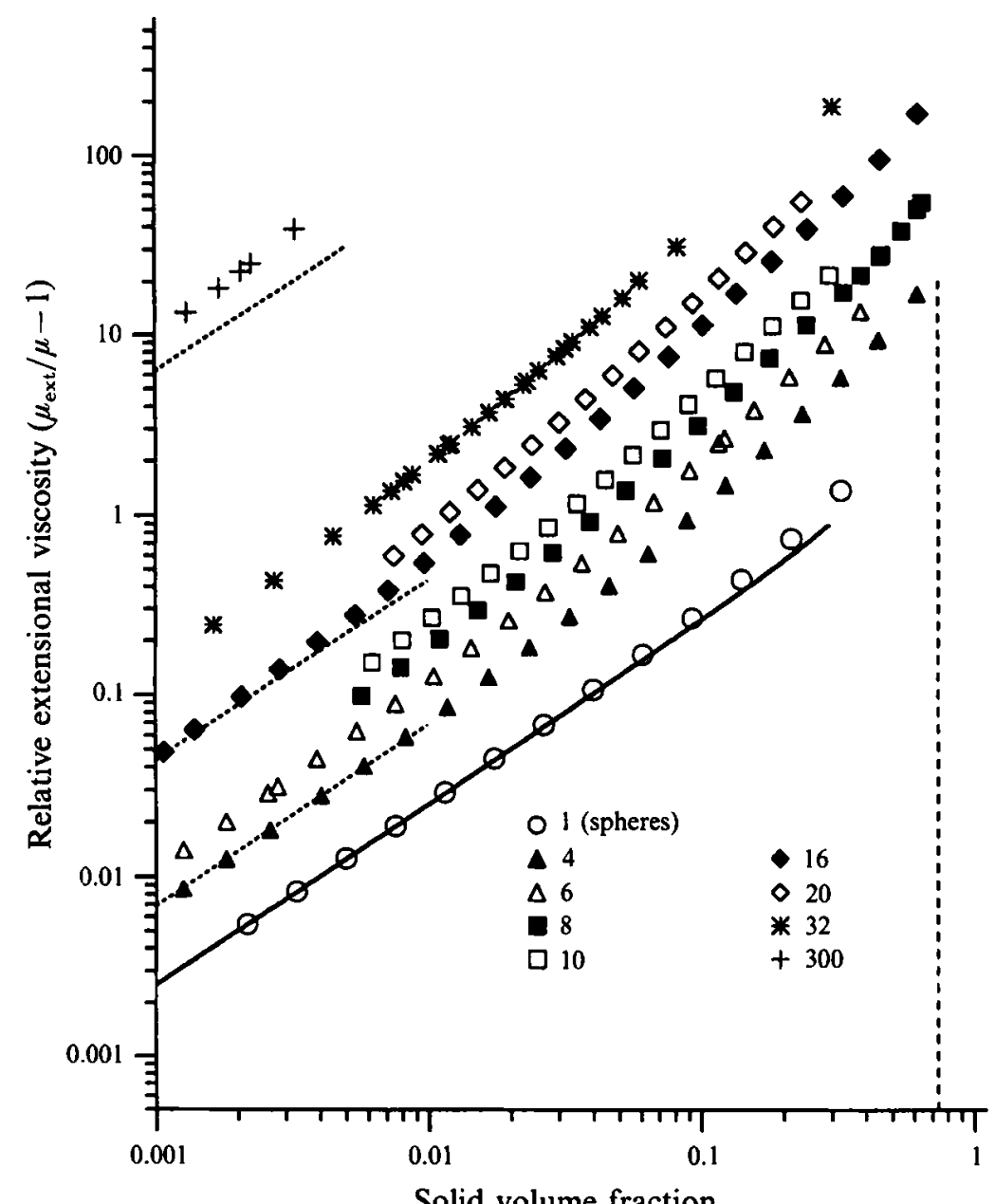

FIGURE 8. Extensional viscosity of efc-lattices of prolate spheroids. Lubrication interactions have been suppressed. The solid line represents the expansion due to Zuzovsky et al. (1983) for suspensions of spheres. The dilute limit expansions for $r_{p}=4,16$ and 300 are shown as dotted lines.

with $r_{p}^{-2}=1-e^{2}$. These asymptotic forms for the coefficients $\eta$ (in the double limit $n_{\phi} \rightarrow 0$ and $\left.r_{p} \rightarrow \infty\right)$ are remarkably accurate for spheroids with $r_{p}>5$ (Kim 1986). Figure 7 shows that they also provide rather good estimates of $\eta_{\perp}$ and $\eta_{\|}$even for quite concentrated systems, since the apparent viscosity is well fitted by the line $\mu_{\mathrm{app}}=\mu(1+2 \phi)$ for $\phi$ up to about $10 \%$ if $r_{p} \geqslant 4 . \dagger$ Note that this expression for $\mu_{\mathrm{app}}$ is only valid for flows which do not have extensional components in the direction of the rod axis (i.e. $E^{\infty}: d d=0$ ) since $\eta_{T}$ contributes otherwise. The coefficients $\eta_{\|}$and $\eta_{\perp}$ are indistinguishable on the plot, but a comparison of the numerical value shows that $\eta_{\|}<\eta_{\perp}$ at low concentrations, and vice-versa at high volume fractions.

A look at $\mu_{\text {ext }}$ in figure 8 reveals a richer dependence on concentration and aspect ratio. The dramatic augmentation of the extensional viscosity which can be achieved by adding less than 1000 p.p.m. of fibrous material to the dispersion is obviously of

+ Similarly, it has been noted by others that the Einstein correction to the viscosity of a suspension of spheres, $\mu_{\text {eff }}=\mu\left(1+\frac{5}{2} \phi\right)$, holds well up to $10 \%$ by volume. 
tremendous practical importance (Mewis \& Metzner 1974). Note also the strong effect of the aspect ratio on the resistance of the suspension to stretch. For comparison, the extensional viscosity of a face-centred cubic-lattice of spheres has also been shown, together with the expansion to $O\left(\phi^{\frac{10}{3}}\right)$ due to Zuzovsky, Adler \& Brenner (1983). The deviation of the simulation results from this theoretical prediction at the higher volume fractions is real, as supported by the exact calculations of Nunan \& Keller (1984), with which our data agree very well. The asymptotic formulae obtained from $(5.5 a)$, $\mu_{\mathrm{ext}}=\mu\left(1-2 r_{p}^{2} \phi \alpha_{5}\right)$, have also been pencilled in for spheroids of aspect ratio 4,16 , and 300 . This shows that dispersions containing $0.1 \%$ by volume of short rods may be called dilute, but that hydrodynamic interactions cause significant deviations from dilute suspension behaviour for spheroids of aspect ratio 300 at the same concentration. Indeed, as first suggested by Batchelor (1971), the relevant measure of concentration for a fibrous dispersion is $n_{\phi} a^{3}$ in problems for which hydrodynamic interactions are dominant. This is roughly the volume 'fraction' of the spheres which circumscribe the rods (the apostrophes emphasizing that this fraction may exceed unity). For instance, it is about 20 for the $0.1 \%$ dispersion of spheroids of aspect ratio 300 cited above.

In 1990, Shaqfeh \& Fredrickson calculated the hydrodynamic stress in a suspension of rods by evaluating the first and dominant term in a diagrammatic series representing multiple scattering events of the momentum propagator in a fibrous dispersion. Their rigorous treatment confirms Batchelor's main findings, and extends them to arbitrary orientation distributions. (Batchelor 1971 restricted his analysis to dispersions of parallel fibres.) The analysis rests, however, on the assumption that the locations of the particles are uncorrelated. Nevertheless, it is instructive to examine our numerical results in the light of their theory. We have therefore recast the data as shown in figure 9, where we plot the inverse of the stresslet component $\boldsymbol{S}: \boldsymbol{d d}$ for uniaxial extension about the $z$-axis of an efc-lattice, versus the logarithm of the volume fraction. (By definition, see (5.3), the ordinate is equal to $\left(8 \pi a^{3} \mu \eta_{T}\right)^{-1}=\left[\left(\mu_{\text {ext }}-\mu\right) / n_{\phi}\right]^{-1}$.) For aligned identical slender ellipsoids of revolution, Shaqfeh \& Fredrickson (1990) obtained

$$
\frac{\mu_{\mathrm{ext}}}{\mu}-1=\frac{8 \pi a^{3} n_{\phi}}{3 \ln (1 / \phi)}\left(1-\frac{\ln \ln (1 / \phi)}{\ln (1 / \phi)}-\frac{1.4389}{\ln (1 / \phi)}\right)+O\left(\frac{n_{\phi} a^{3}}{\ln ^{3}(1 / \phi)}\right) .
$$

They also proved that the inverse dependence on $\ln (\phi)$ in this expression is intimately related to the occurrence of hydrodynamic screening in suspensions of rods, as intuitively hypothesized by Batchelor (1971). Physically, the rigid bodies oppose any disturbance which changes direction over distances much shorter than their largest dimension, even if they are neutrally buoyant and exert no net force on the fluid; i.e. the Fourier components of incident velocity fields with a wavelength of $o(a)$ are effectively screened by freely suspended rods. This opposition to the fluid motion raises the viscosity of the dispersion. It is well known that the screening length $\zeta$ (i.e. the characteristic lengthscale for the decay of velocity disturbances) varies as $\phi^{-\frac{1}{2}}$ in random dispersions, but that it is shorter ranged, of $O\left(\phi^{-\frac{1}{3}}\right)$, for simple lattices. This also holds in fibrous suspensions, and Shaqfeh \& Fredrickson (1990) report for random dispersions of rods that $\zeta \sim\left(n_{\phi} a\right)^{-\frac{1}{2}}$. To account for the difference between the screening lengths in ordered and disordered dispersions, we naively rescale (5.6), which was derived using the assumption that the positions of the rods are uncorrelated, by a factor $\frac{2}{3}$. This is obviously ad hoc, since there is no reason to believe that the second and third terms in the expansion (5.6) should scale like the first. Yet we take the remarkably good agreement with our simulation results noted in figure 9 to indicate that this reasoning is at least qualitatively correct. The transition from a regime in 


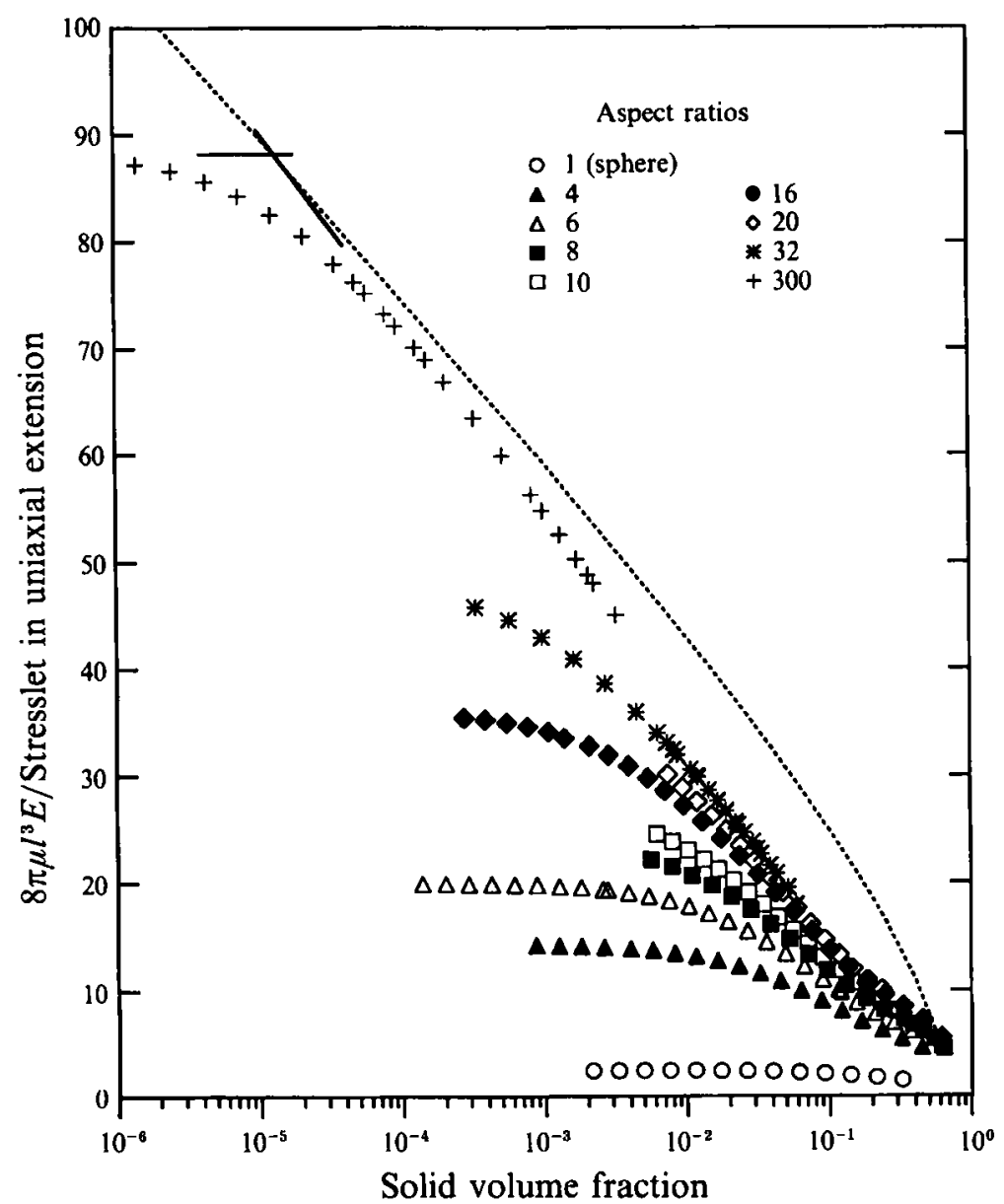

FIGURE 9. Verification of hydrodynamic screening in efc-dispersions of spheroids. The dotted line represents equation (5.6) multiplied by $\frac{3}{2}$ to account for the perfect correlation of the centres of mass in this system.

which the mean stresslet is independent of volume fraction (as it should be for dilute systems) to a concentration range where $S: d d \sim(\ln (1 / \phi))^{-1}$ is clearly apparently and is consistent with the idea of screening. Quite fortuitously, the break point in figure 9 corresponds almost exactly to $\frac{4}{3} \pi n_{\phi} a^{3}=O(1)$, as predicted by theory.

\section{Dependence on the crystal geometry}

One may wonder how representative the properties of an efc-lattice are of other crystal geometries. The fact that the maximum packing attainable for slender bodies depends so critically on the arrangement hints that the microstructure matters much more for spheroids than for spheres. For instance, we noted earlier that an arrangement of parallel impenetrable spheroids on a simple cubic lattice has a density of at most $\pi / 6 r_{p}^{2}$, while efc structures accept volume fractions as high as $\pi / 3 \sqrt{ } 2$. This is a considerable difference even for moderate aspect ratios. In contrast, body-centred cubic cells of sphere may be filled up to $\sqrt{ } 3 \pi / 8$, which is not very different from the densest (face-centred or hexagonally close packed) structure. We explained in $\S 2$ how 


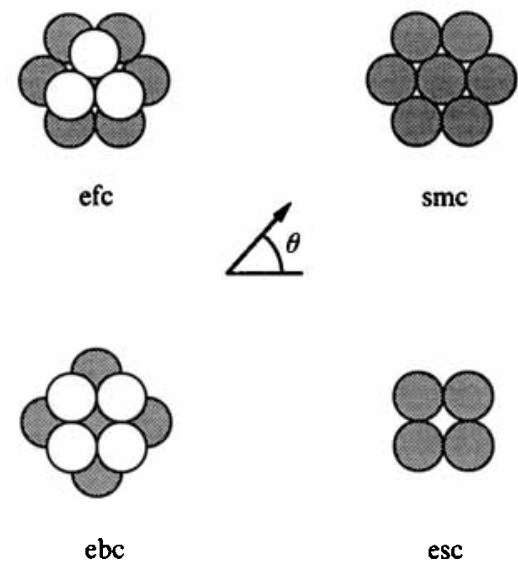

Figure 10. Projection of the lattice geometry onto the close packed planes (i.e. along the spheroidal axis). The white and shaded particles are in adjacent layers in the crystal.

to construct regular close-packed crystals of arbitrary, uniform spheroids by stretching the corresponding unit cell for spheres by a factor equal to the aspect ratio, and stressed that this transformation does not affect the packing fraction. We also showed that an isotropic dilution of this dense microstructure results in uninteresting 'twodimensional' configurations as a result of the anisotropy of the unit cell at maximum packing, and introduced the alternative procedure of expanding the lattice by keeping the surface-to-surface separation between nearest neighbours the same in three nonplanar directions. This guarantees that the dispersion possesses as much 'threedimensional character' as is compatible with the chosen concentration. We apply this technique to four types of microstructures in what follows to investigate the impact of the detailed crystal geometry on the macroscopic properties. One lattice will also be expanded isotropically in order to judge the effect of the dilatation technique on the transport coefficients of ordered configurations. We shall primarily focus our discussion on the relation between the stress and the rate of strain because of its primary interest in the rheology of rod dispersions. Furthermore, this characteristic can be estimated reliably with Stokesian dynamics (keeping only the effects of the force, torque and stresslet exerted by the spheroids on the fluid), and is relatively insensitive to the number of particles used in the unit cell (Phillips, Brady \& Bossis 1988; Ladd 1990).

Three of the five lattices considered here (figure 10) are obtained by distorting elementary modules of spheres, namely:

(i) the efc-structure of the previous section, with maximum packing $\pi / 3 \sqrt{ } 2$, obtained by stretching a face-centred unit cell of spheres along (111),

(ii) the ebc-configuration, constructed by expanding the same lattice along (001). This obviously has the same maximum density as the efc-variant. For $r_{p}=1$, both reduce to the face-centred cubic geometry, and are indistinguishable. (They then become descriptions of the same packing in two different frames of reference.) Note that the ebc-crystal can be thought of as a staggered arrangement of spheroids placed on a square grid, just as the efc-lattice consists of stacks of particles distributed on a hexagonal mesh. In this sense, the ebc-lattice is related to the body-centred crystal of spheres, hence the acronym,

(iii) the esc-structure, obtained from a simple cubic cell of spheres by stretching it along (001). This geometry resembles the ebc-lattice, but the stacks of spheroids are now facing each other instead of being offset by half a mesh size for a tighter packing. 
The gap width between the layers (i.e. the distance between the poles of two spheroids in adjacent stacks) is equal to the smallest surface-to-surface separation within the sheets, consistent with the convention used throughout this work (except for the iscstructure discussed below). The maximum density of this crystal is $\frac{1}{6} \pi$.

A fourth crystal is built so as to bear the same relation to the efc-lattice as the escconfiguration vis-ä-vis the ebc-lattice:

(iv) in the smc-structure, honeycomb layers of spheroids are stacked directly on top of one another in a manner similar to the square arrays of particles in the escconfiguration. This packing is somewhat less efficient than that achieved in the efc and ebc crystals, and accommodates at most $\pi /(3 \sqrt{ } 3)$. The abbreviation smc was chosen because this geometry is reminiscent in a crude way of the molecular arrangement in a smectic $A$.

The fifth and last family of crystals to be defined is

(v) the isc-lattice, which is identical to the esc-configuration when the particles touch (i.e. it is a stretched simple cubic cell at closest packing), but is then expanded to lower volume fractions by dilating isotropically, instead of respecting the usual convention which stipulates that the surface-to-surface separation is the same in at least three nonplanar directions.

In order to accentuate the differences in the stress response of the various crystals, the data are presented in terms of the average stresslet $\eta$ per particle (appropriately nondimensionalized) rather than the apparent viscosity. As shown in the previous section, these quantities are related by $\mu_{\mathrm{app}}=\mu\left(1+6 r_{p}^{2} \phi \eta\right)$. Although we shall use the symbols $\eta_{T}, \eta_{\|}$and $\eta_{\perp}$ to denote the flow conditions illustrated in figure 6, the tensor $\boldsymbol{R}_{\Sigma E}$ does not in general have the form (5.3). Figure 11 summarize our findings for dispersions spheroids of aspect ratio 8 . The coefficient $\eta_{\perp}$ is obviously the least sensitive to the detailed features of the crystal geometry. A closer look reveals however, that the microstructures with the smallest $\eta_{\perp}$ at a given volume fraction (i.e. the lattice which offers the least resistance to shear flows having a velocity gradient parallel to the direction of alignment, see figure 6) display the highest extensional viscosity (i.e. the largest $\eta_{T}$ ). In fact, the ordering of the lattices according to $\eta_{\perp}$ is exactly the reverse of the order obtained using $\eta_{r}$. This correlation is not surprising since both coefficients are associated with flows straining the spheroids in a plane containing their axes.

The staggered configurations (ebc and efc) display the largest $\eta_{\perp}$ (figure $11 a$ ). This reflects the steric hindrance to rotation brought about by interlacing the stacks of spheroids. This reduced orientational freedom (confirmed by comparing the 'sedimentation rates' $V_{\perp}^{\text {ro }}$ of the various crystals (data not shown)) increases the stress response of the dispersion to any shearing flow which induces a rotation in a plane containing $d$. This phenomenon is appropriate to non-spherical particles, for which a strong coupling exists between the stress and the rotation rate (i.e. $\boldsymbol{R}_{S \Omega} \neq 0$ ). Indeed, a close inspection of the grand mobility tensor clearly demonstrates that the origin of the larger $\eta_{\perp}$ for staggered configurations resides in the larger $\boldsymbol{M}_{\Omega S}$ for these systems. This effect is more pronounced for the ebc-lattice than for the efc-geometry because each particle has eight out-of-plane neighbours which hinder its rotation in the former crystal (and four in-the-plane neighbours which reinforce its motion), as opposed to six out-of-plane neighbours and six in the same layer for the efc-lattice. Finally, the isogeometry, which leaves the largest gaps between the layers, clearly has the lowest $\eta_{1}$, in accordance with the arguments above.

Despite the aforementioned correlation between $\eta_{\perp}$ and $\eta_{T}$, the physical mechanism responsible for the decreased extensional viscosity in staggered lattices cannot be traced to the coupling between the rotation rate and the stress $\left(\boldsymbol{M}_{\Omega S}\right)$, since this is 


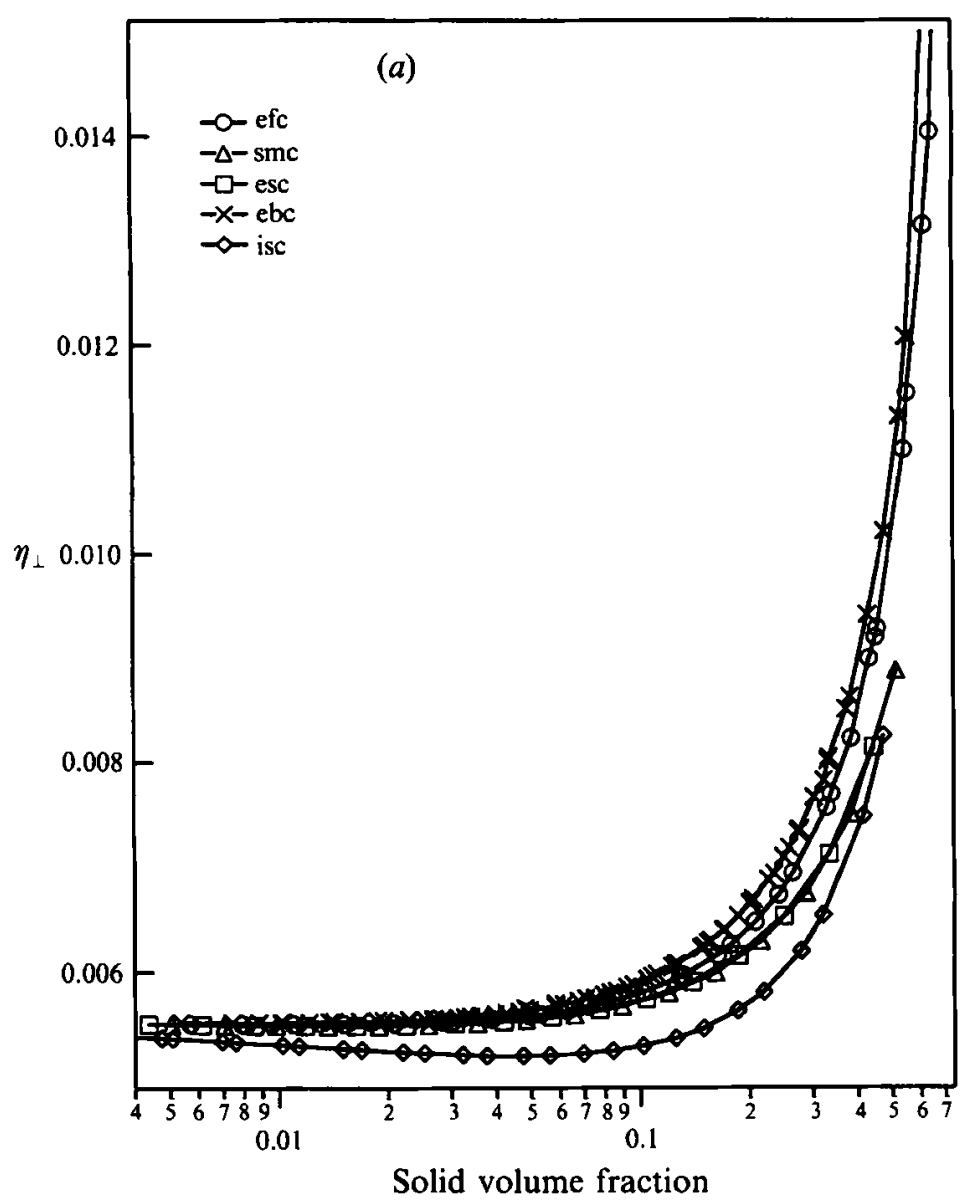

FIGURE 11 (a). For caption see p. 495.

irrelevant for uniaxial extension. (This can best be seen by observing that the spheroid does not reorient when the principal direction of strain coincides with its axis.) Instead, the stronger resistance to axisymmetric extension of the smc- and esc-configurations is directly related to a reduction of the diagonal elements of $\boldsymbol{M}_{E S}$ compared to their values for efc- and ebc-lattices at the same volume fraction. The analysis by Shaqfeh \& Fredrickson (1990) and the qualitative argument justifying the cell model used by Batchelor (1971) to calculate the extensional viscosity of a suspension of uniformly aligned rods emphasize the importance of the smallest distance between the fibres (measured perpendicular to the particles' axes, and not between their geometric centres), since this is the lengthscale for hydrodynamic screening. This is the smallest for isc-crystals, which have to compensate for the loose stacking of the layers by packing the spheroids more tightly within these layers. This lattice correspondingly has the highest $\eta_{T}$.

The response of the dispersions to simple shear in the $(x, y)$-plane, finally, depends mostly on the shortest distance between the fibres along the principle directions of the strain (i.e. along the compressional and extensional axes of the flow). These distances are listed in table 1 for all lattices at $\phi=0.1$, and can be seen to correlate well with $\eta_{\|}$. For crystals with hexagonal rather than square symmetry in the plane of shear (smc and efc), the separation given is the centre-to-centre distance between nearest 


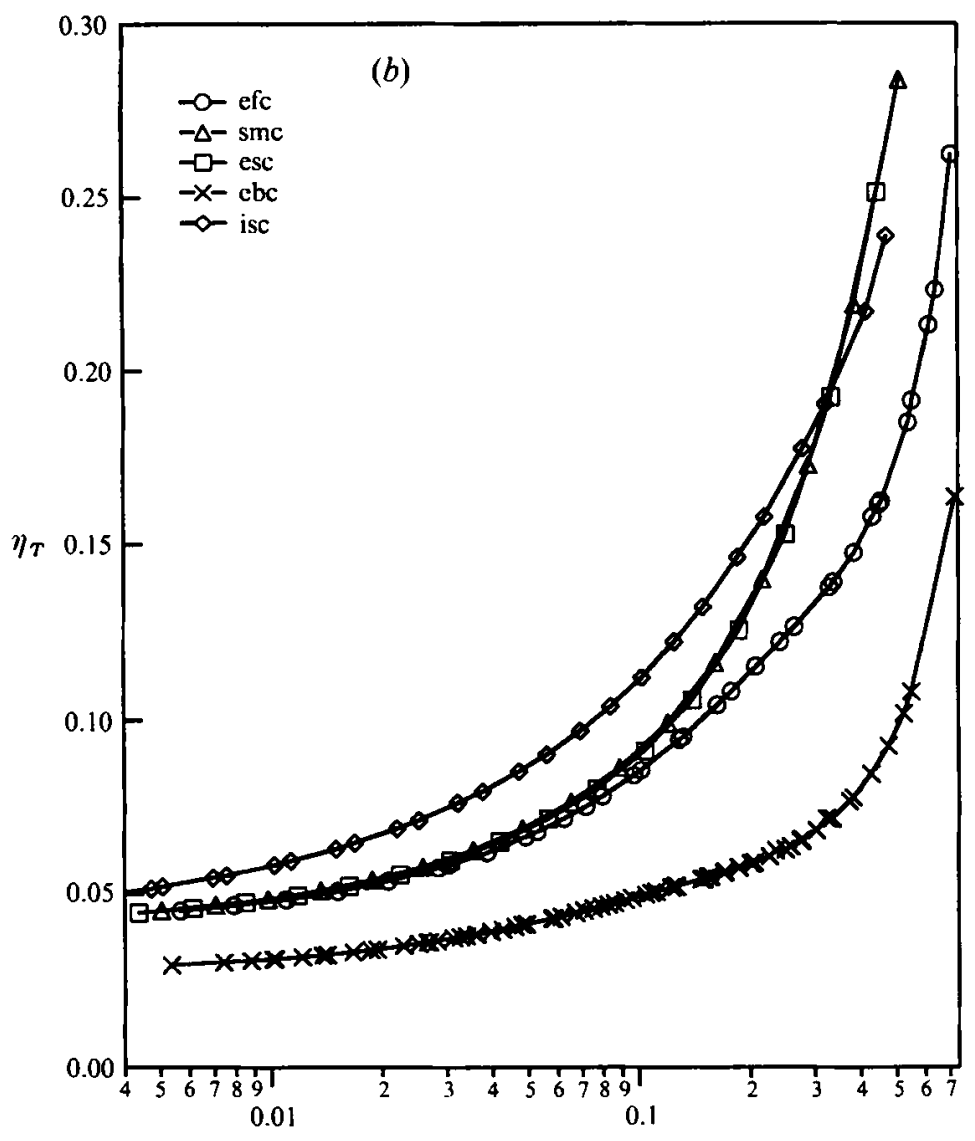

Solid volume fraction

FIGURE $11(b)$. For caption see facing page.

\begin{tabular}{ccccccccc}
\hline & efc & smc & esc & esc & ebc & ebc & isc & isc \\
$\theta$ & $\frac{1}{12} \pi$ & $\frac{1}{12} \pi$ & $\frac{1}{4} \pi$ & 0 & $\frac{1}{4} \pi$ & 0 & $\frac{1}{4} \pi$ & 0 \\
$\eta_{1}\left(\times 10^{3}\right)$ & 5.99 & 6.06 & 7.57 & 5.03 & 7.08 & 5.06 & 9.41 & 5.07 \\
$r / a$ & 0.592 & 0.569 & 0.535 & 0.757 & 0.570 & 0.806 & 0.429 & 0.606
\end{tabular}

TABLE 1. Shortest centre-to-centre distance along the principal directions of strain of a simple shear flow for several crystal geometries at $\phi=0.1\left(r_{p}=8\right)$. The flow direction lies perpendicular to the axis of the particles. $r / a$ is the shortest distance between the centres of the spheroids in the given configuration, measured at $\frac{1}{4} \pi$ or $\frac{3}{4} \pi$ relative to the direction of flow in the plane of shear (i.e. along the compressional and extensional axes of the straining field). As always, $a$ denotes the particle halflength. The angle $\theta=0$ if the undisturbed velocity lies along the shortest vector of the lattice (figure 10). See also figure 11.

neighbours. The spacing of the particles, averaged along both the compressional and extensional axes of the shear, will certainly be larger for these configurations, which explains why the efc- and smc-lattices display lower apparent shear viscosities than suggested by the distances of table 1 . 


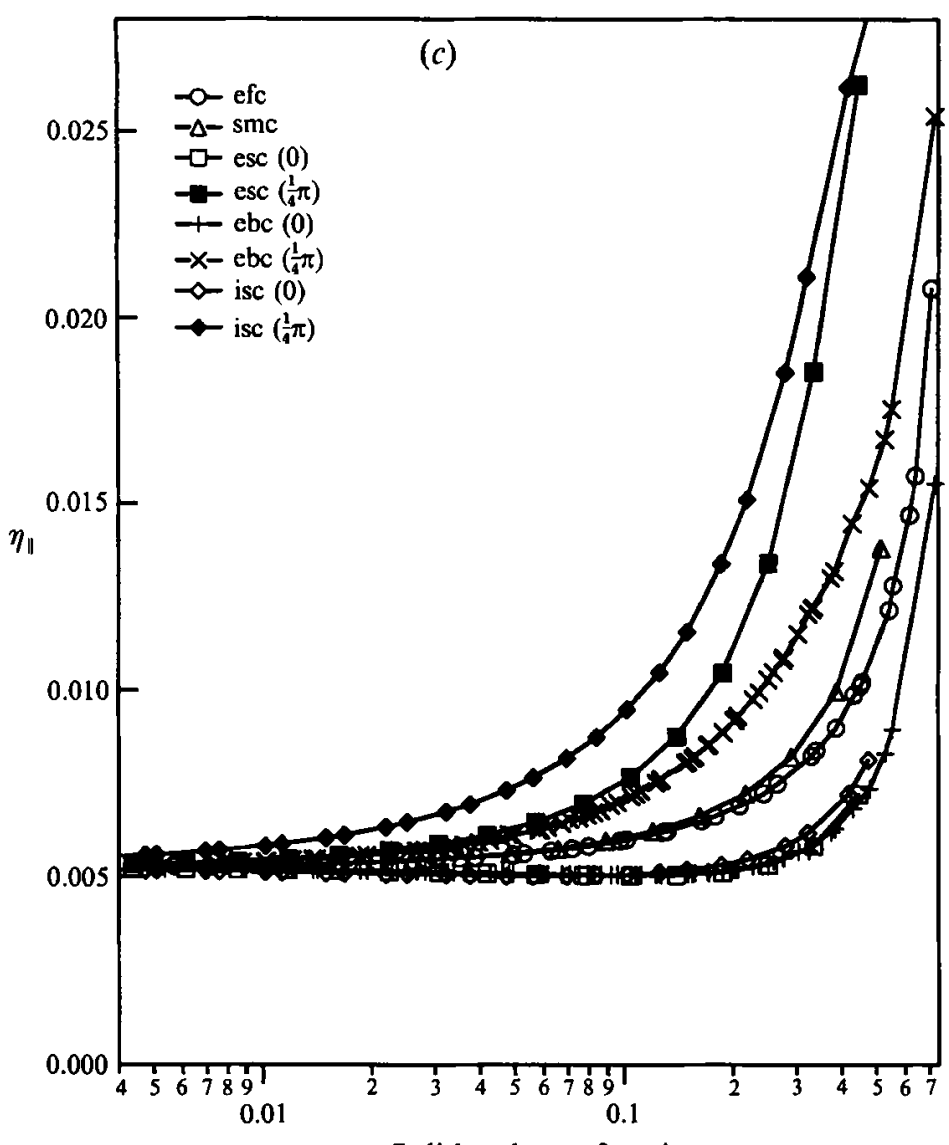

Solid volume fraction

Figure 11. Coefficients $(a) \eta_{1},(b) \eta_{T},(c) \eta_{1}$ of the relation (5.3) between the hydrodynamic stress and the rate of strain for a variety of crystalline configurations of spheroids of aspect ratio 8 . The number in parentheses in the legend of $(c)$ indicates the angle $\theta$ between the shortest basis vector of the lattice and the direction of the shear flow (figure 10).

\section{Conclusions}

An extended version of Stokesian dynamics (Claeys \& Brady 1993a,b) was used to study the hydrodynamic transport properties of crystals of parallel spheroids over the full concentration range from zero to closest packing ( $74 \%$ by volume). These structures possess long-ranged orientational order, and can be viewed as models of liquid crystalline domains in that sense. The concentration dependence of the sedimentation rate reveals cooperative viscous interactions enhancing the collective rotational diffusion coefficient $V_{\perp}^{r o}$. This effect is lost in fibrous media, and all hindered diffusivities decrease monotonically with density. The variation of the extensional viscosity of efc-lattices with volume fraction supports the concept of hydrodynamic screening in suspensions of force-free rods, as proposed by Batchelor (1971) and by Shaqfeh \& Fredrickson (1990). The effect of the detailed microstructural features was also investigated by calculating the rheological data for five different lattices of aligned ellipsoids. Two geometric characteristics seem to influence the stress response of the crystals the most: the shortest distance between the fibres, and the way layers of particles are stacked to form the dispersion (in particular, whether the sheets of spheroids are 'interpenetrating'). This study illustrates the role of microstructure in 
determining the properties of suspensions, and demonstrates the effectiveness of Stokesian dynamics for simulations of many-body low-Reynolds-number problems involving rod-like particles.

\section{Appendix A. Basis vectors and symmetry properties of crystalline configurations of spheroids}

In the (theoretical) rheology of suspensions, the value and accuracy of novel numerical methods is often ascertained by computing the concentration dependence of the transport properties of face-centred cubic lattices of spheres (Zick \& Homsy 1982; Zuzovsky et al. 1983; Nunan \& Keller 1984; Brady et al. 1988; Ladd 1988). We propose that the regular arrays of prolate spheroids discussed in this article, in particular the efc-crystal, can serve a similar purpose for dispersions of elongated particles. Their geometry is unambiguously defined, and among the simplest which accept the maximum + packing fraction $\pi / 3 \sqrt{ } 2$ while retaining a fully three dimensional character at lower concentrations. Their high degree of symmetry allows general predictions to be made concerning their properties and may make them amenable to some kind of analytical treatment against which present and future simulations can be tested for consistency. (The calculation of the dielectric constant of a regular array of prolate spheroids (Lam 1990) offers hope that the advent of new mathematical tools will soon allow a closed-form expression for the extensional viscosity of efc-crystals to be derived, for instance.) We therefore consider it appropriate to carefully characterize these lattices here, and to discuss some of their properties. A few tables of representative data can be obtained from the authors or the editor.

The close-stacked efc-lattice is derived from a face-centred crystal of touching spheres by stretching it by a factor equal to the aspect ratio in a direction perpendicular to the hexagonally packed planes (i.e. along the (111)-diagonal of the cubic cell) (figure 1). This transformation maps spheres onto ellipsoids without altering the volume fraction of the array. We shall refer to the direction in which the spheroids point (which is also the direction of stretch) as the $\boldsymbol{e}_{3}$-axis, and introduce an orthonormal Cartesian coordinate system $\left(e_{1}, e_{2}, e_{3}\right)$, with origin at the core of a particle, such that $e_{1}$ lies along a line of centres (figure 12a). Non-dimensionalizing distances by the length $a$ of the major semi-axis of the spheroids, the matrix $\boldsymbol{B}$ defining the lattices can be chosen as

$$
\boldsymbol{B}=\left(\begin{array}{ccc}
2 / r_{p} & 1 / r_{p} & 0 \\
0 & \sqrt{ } 3 / r_{p} & \frac{2}{3} \sqrt{ } 3 / r_{p} \\
0 & 0 & 2 \sqrt{ } \frac{2}{3}
\end{array}\right) .
$$

The three columns of $B$ form a set of basis vectors $\left\{\boldsymbol{b}_{1}, \boldsymbol{b}_{2}, \boldsymbol{b}_{3}\right\}$ for the crystals, i.e. the location of the geometric centres $\boldsymbol{x}_{p}$ of all particles belonging to the efc-crystal can be written uniquely as $\boldsymbol{x}_{p}=\boldsymbol{B} \cdot \boldsymbol{z}_{p}$, with $\boldsymbol{z}_{p}$ a set of three integer coordinates. The volume of a spheroid is $\frac{4}{3} \pi / r_{p}^{2}$, and that of the unit cell, $|\boldsymbol{B}|=4 \sqrt{ } 2 / r_{p}^{2}$, giving a packing fraction $\phi=\pi /(3 \sqrt{ } 2)$, the theoretical regular close-packed limit for dispersions of ellipsoids.

In order to study 'face-centred' suspensions at concentrations other than the maximum, we proposed to expand the crystal from closest packing in such a way that

+ This statement has not yet been mathematically proven. Even for spheres, it has not been demonstrated rigorously in three dimensions that the maximum volumetric concentration compatible with the mutual impenetrability of the particles is $\pi / 3 \sqrt{ } 2$, the density of a close packed face-centred lattice (Gruber \& Lekkerkerker 1987), but this is generally accepted. The problem is even more complex for prolate spheroids, as there is an additional degree of freedom associated with each particle, yet the same maximum packing fraction is usually assumed (Frenkel \& Mulder 1985). 
(a)

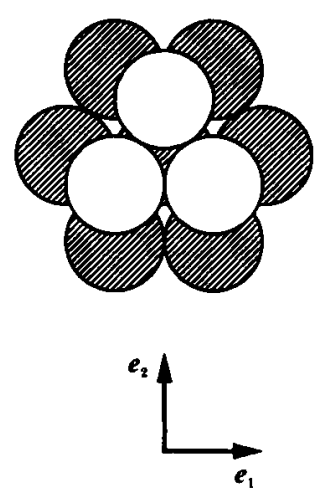

(b)

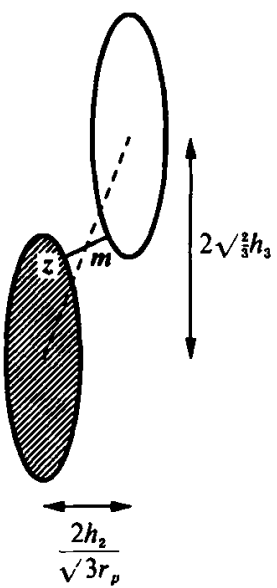

Figure 12. Definition of an efc-crystal of spheroids. (a) Projection onto the $\left(e_{1}, e_{2}\right)$-plane. The spheroidal axis (in the $e_{3}$-direction) points out of the paper. The white particles are stacked on top of the shaded ones. The layer below is staggered with respect to both of the layers shown, in the customary 'ABCABC'-repeat structure of face-centred crystals. (b) Cross-section of the dispersion in the $\left(e_{2}, e_{3}\right)$-plane.

the minimum surface-to-surface separation $\delta$ between nearest neighbours is the same in three non-planar directions. This procedure maintains the 'three-dimensional' character of the dispersion at all volume fractions, and is taken as the definition of an efc-lattice. The most obvious alternative, an isotropic dilation of the crystal, preserves the anisotropy present in the unit cell at closest packing, i.e. $b_{3} \cdot e_{3} / b_{1} \cdot e_{1}=\sqrt{ } \frac{2}{3} r_{p}$. For slender particles, this rapidly degenerates into rather uninteresting 'two-dimensional' structures consisting of sheets of hexagonally packed spheroids, separated by wide gaps of fluid. Efc-crystals, on the other hand, converge to face-centred cubic lattices at infinite dilution, independent of the aspect ratio of the particles. For a chosen $\delta$, the appropriate expansion factors $h_{i}$ in each of the three directions $e_{i}$ can be determined as follows. It is easy to see from the hexagonal pattern in the $\left(e_{1}, e_{2}\right)$-plane (figure 12a) that

$$
\delta=\left(h_{1}-1\right) 2 / r_{p}
$$

and that $h_{1}=h_{2}$. To find $h_{3}$, imagine a cross-section of the dispersion along the $\left(e_{2}, e_{3}\right)$ plane, and isolate for consideration two neighbouring particles in adjacent hexagonal layers (figure $12 b$ ). Label the midpoint of the line joining their centres

$$
m=h_{2} /\left(\sqrt{ } 3 r_{p}\right) e_{2}+\sqrt{ } \frac{2}{3} h_{3} e_{3},
$$

and call $z$ the point of closest approach on the spheroid at the origin. Since $z$ belongs to the surface of the ellipsoid, we know that

$$
\left(z \cdot e_{3}\right)^{2}+r_{p}^{2}\left|z \wedge e_{3}\right|^{2}=1 \text {. }
$$

The normal at that point is parallel to $\left(z \cdot e_{3}\right) e_{3}+r_{p}^{2}\left[z-\left(z \cdot e_{3}\right) e_{3}\right]$, which equals $\left(z \cdot e_{3}\right) e_{3}+r_{p}^{2}\left(z \cdot e_{2}\right) e_{2}$ since $z$ lies in the $\left(e_{2}, e_{3}\right)$-plane. By symmetry, it must pass through $m$. Hence, letting $z \cdot e_{3}=z_{3}$ and $z \cdot e_{2}=z_{2}$,

$$
\frac{(m-z) \cdot e_{3}}{(m-z) \cdot e_{2}}=\frac{\sqrt{ } \frac{2}{3} h_{3}-z_{3}}{h_{2} /\left(\sqrt{ } 3 r_{p}\right)-z_{2}}=\frac{z_{3}}{r_{p}^{2} z_{2}}
$$


The shortest distance between the ellipsoids is measured along this normal, so that also

$$
\frac{1}{4} \delta^{2}=\left(\sqrt{\frac{2}{3}} h_{3}-z_{3}\right)^{2}+\left(\frac{h_{2}}{\sqrt{3} r_{p}}-z_{2}\right)^{2} .
$$

Squaring (A 4) and eliminating $z_{3}$ using (A 3), we obtain, after a little rearrangement of (A 5), that

$$
\frac{1}{4} \frac{\delta^{2} z_{2}^{2}}{\left(h_{2} /\left(\sqrt{ } 3 r_{p}\right)-z_{2}\right)^{2}}=\frac{1}{r_{p}^{4}}+\frac{r_{p}^{2}-1}{r_{p}^{2}} z_{2}^{2} .
$$

The right-hand side has a slope of less than 1 when plotted as a function of $z_{2}^{2}$, and hence (A 6) can be used to generate a stable iterative scheme for $z_{2}$ as $\lim _{k \rightarrow \infty} z_{2}^{(k)}$ with

$$
\begin{gathered}
\frac{1}{4} \frac{\delta^{2}\left(z_{2}^{(k+1)}\right)^{2}}{\left(h_{2} /\left(\sqrt{ } 3 r_{p}\right)-z_{2}^{(k+1)}\right)^{2}}=\frac{1}{r_{p}^{4}}+\frac{r_{p}^{2}-1}{r_{p}^{2}}\left(z_{2}^{(k)}\right)^{2}=l^{(k)}, \\
z_{2}^{(k+1)}=\frac{h_{2} /\left(\sqrt{ } 3 r_{p}\right)}{1+\delta /\left(2\left(l^{(k)}\right)^{\frac{1}{2}}\right)} .
\end{gathered}
$$

From $z_{2}$, it is trivial to calculate $z_{3}$ and $h_{3}$. The construction of the other four lattices defined in the text is fairly simple, and an appropriate set of basis vectors can be found as explained above for the efc-structure.

From figure 12(a), it is apparent that the efc-lattice is invariant under a set of coordinate transformations. These symmetry elements imply that the material properties of the crystal must obey certain rules. Consider for instance a fourthrank tensor $\boldsymbol{Y}$, which is a function of the particle configuration only, and denote $\boldsymbol{\gamma} \odot \odot^{4} e_{l} \boldsymbol{e}_{k} \boldsymbol{e}_{j} \boldsymbol{e}_{i}$ by $Y_{i j k l}$. The reflection symmetry about the origin then stipulates that $Y_{i j k l}=0$ whenever an odd number of the coordinate labels 1, 2, or 3 appear in the indices $i j k l$. Assuming that $\boldsymbol{Y}$ is symmetric in its first and last two indices and that $Y_{i j k l}=Y_{k l i j}$ (a consequence of Lorentz' reciprocal theorem in the case of $\boldsymbol{R}_{\Sigma E}$ ), this reduces the number of independent coefficients characterizing the material property to nine. The invariance of the crystal structure under a rotation by $\frac{1}{3} \pi$ in the $\left(e_{1}, e_{2}\right)$-plane imposes the additional relations

$$
\begin{gathered}
Y_{1133}=Y_{2233}, \quad Y_{1313}=Y_{2323}, \quad Y_{1111}=Y_{2222}, \\
Y_{1111}=Y_{1122}+2 Y_{1212} .
\end{gathered}
$$

These can be shown by standard techniques to hold for every geometry that is indifferent to a rotation in the $\left(e_{1}, e_{2}\right)$-plane, except if it concerns $90^{\circ}$ turns (or the special cases $0^{\circ}$ and $180^{\circ}$ ). The fourth-rank tensor $Y$ is now fully determined by only five components. If furthermore we require tracelessness in its first and last two indices, only three independent elements remain. In $\boldsymbol{R}_{\Sigma E}$, we called these three coefficients $\eta_{T}$, $\eta_{\|}$and $\eta_{\perp}$ (see (5.3)). For cubic face-centred crystals, one more invariance relation exists, and the number of independent coefficients drops to two (Nunan \& Keller 1984). Both the efc- and smc-lattices satisfy the symmetries used in this paragraph and their stress/rate-of-strain coupling is consequently characterized by three constants. The ebc-, esc- and isc-dispersions however, must be rotated by $\frac{1}{2} \pi$ rather than $\frac{1}{3} \pi$ to return to the same orientation. This invalidates (A $8 b$ ) so that four components are needed to specify $\boldsymbol{R}_{\Sigma E}$ for these configurations. 


\section{Appendix B. Some hydrodynamic transport coefficients for crystals of spheroids}

As explained in Appendix A, crystals of spheroids are useful reference configurations whose properties serve as benchmarks to test the accuracy of present and future simulation methods. We have therefore collected a few results on the hydrodynamic transport properties of orderly arrangements of spheroids in tables 2-12, which because of their detail are not reproduced here, but are available either from the authors or the Journal of Fluid Mechanics Editorial Office.

We first discuss the relation $\boldsymbol{R}_{\Sigma E}$ between the hydrodynamic stress and the rate of strain. Owing to the tracelessness and symmetry of $\boldsymbol{E}$ and $\boldsymbol{S}$, this tensor is indeterminate. Following the usual convention, we define $\boldsymbol{R}_{\Sigma E}$ uniquely by specifying that it, too, must be traceless and symmetric in its first and last two indices. This allows one to compact it into a $5 \times 5$ matrix $Y$ such that

$$
\left(\begin{array}{c}
\Sigma_{11} \\
\Sigma_{22} \\
\Sigma_{12} \\
\Sigma_{23} \\
\Sigma_{31}
\end{array}\right)=8 \pi a^{3} \mu r \cdot\left(\begin{array}{c}
E_{11}-E_{33} \\
E_{22}-E_{33} \\
2 E_{12} \\
2 E_{23} \\
2 E_{31}
\end{array}\right),
$$

with $\mu$ the viscosity of the suspending fluid and $a$ the length of the spheroidal semi-axis. It follows from Lorentz' reciprocal theorem that $\boldsymbol{V}$ is symmetric. It thus contains only 15 independent elements:

$$
\boldsymbol{V}=\left(\begin{array}{lllll}
Y_{1111} & Y_{1122} & Y_{1112} & Y_{1123} & Y_{1311} \\
Y_{1122} & Y_{2222} & Y_{2212} & Y_{2223} & Y_{2231} \\
Y_{1112} & Y_{2212} & Y_{1212} & Y_{1223} & Y_{1231} \\
Y_{1123} & Y_{2223} & Y_{1223} & Y_{2323} & Y_{2331} \\
Y_{1131} & Y_{2231} & Y_{1231} & Y_{2331} & Y_{3131}
\end{array}\right)
$$

For dispersions possessing a 6-fold axis of symmetry $d$, invariance laws imply that three components suffice to characterize $\boldsymbol{Y}$ (Appendix A). In terms of the coefficients $\eta$ introduced in (5.3), and assuming that $d$ lies along the ' 3 ' axis, it is easy to find in this case that

$$
\boldsymbol{Y}=\left(\begin{array}{ccccc}
\frac{1}{3} \eta_{T}+\eta_{\|} & \frac{1}{3} \eta_{T}-\eta_{\|} & 0 & 0 & 0 \\
\frac{1}{3} \eta_{T}-\eta_{\|} & \frac{1}{3} \eta_{T}+\eta_{\|} & 0 & 0 & 0 \\
0 & 0 & \eta_{\|} & 0 & 0 \\
0 & 0 & 0 & \eta_{\perp} & 0 \\
0 & 0 & 0 & 0 & \eta_{\perp}
\end{array}\right) .
$$

By analogy, we set $\eta_{T}=\frac{3}{2}\left(Y_{1111}+Y_{1122}\right), \eta_{\|}=Y_{1212}$ and $\eta_{\perp}=Y_{3131}$ for all configurations in figure 11, even though (5.3) does not hold in general. This definition of $\eta_{T}$ makes it equal to one third of the Trouton viscosity in uniaxial extensional flows along the ' 3 ' axis. For isotropic suspensions, one sees from (B 3) that $\boldsymbol{V}$ has seven non-zero elements, two of which are identical because of symmetry. The six remaining components can be averaged appropriately to obtain a more reliable estimate of the effective viscosity, as done in Claeys \& Brady (1993b).

Lubrication interactions were suppressed in all calculations concerning crystalline dispersions for reasons explained in $\S 3$. We non-dimensionalize the transport properties 
as follows: diffusion coefficients scale with $k T / 8 \pi \mu a^{n} \quad(n=1$ for translational components, $n=3$ for rotary diffusivities), sedimentation rates with $|F| / 8 \pi \mu a$ and their rotational analogs with $|T| / 8 \pi \mu a^{3}$. ( $\boldsymbol{F}$ and $T$ represents the uniformly applied force or torque respectively, $k$ is Boltzmann's constant, and $T$ the absolute temperature.)

\section{REFERENCES}

Allen, M. P., Frenkel, D. \& Talbot, J. 1989 Comput. Phys. Rep. 9, 301-353.

BATChELOR, G. K. 1970 J. Fluid Mech. 41, 545-570.

BATCHELOR, G. K. 1971 J. Fluid Mech. 46, 813-829.

Bitsanis, I., Davis, H. T. \& TirRell, M. 1988 Macromol. 21, 2824-2835.

Bitsanis, I., Davis, H. T. \& Tirrell, M. 1990 Macromol. 23, 1157-1165.

Brady, J. F. \& Bossis, G. 1988 Ann. Rev. Fluid Mech. 20, 111-157.

Brady, J. F., Phillips, R. J., Lester, J. C. \& Bossis, G. 1988 J. Fluid Mech. 195, 257-280.

Chwang, A. T. \& Wu, T. Y.-T. 1975 J. Fluid Mech. 67, 787-815.

Clafys, I. L. \& Brady, J. F. 1989 PhysicoChem. Hydrodyn. 11, 261-293.

Claeys, I. L. \& Brady, J. F. 1993 a J. Fluid Mech. 251, 411-442.

Claeys, I. L. \& Brady, J. F. $1993 b$ J. Fluid Mech. 251, 443-477.

Frenkel, D. 1987 Molec. Phys. 60, 1-20.

Frenkel, D. \& Mulder, B. M. 1985 Molec. Phys. 55, 1171-1192.

Frenkel, D., Mulder, B. M. \& MCTaGue, J. P. 1984 Phys. Rev. Lett. 52, 287-290.

Gruber, P. M. \& LeKKERKERKER, C. G. 1987 Geometry of Numbers. North-Holland.

Hasimoto, H. 1959 J. Fluid Mech. 5, 317-328.

HiNCH, E. J. 1972 J. Fluid Mech. 54, 423- 425.

KIM, S. 1986 Intl J. Multiphase Flow 12, 469-491.

Ladd, A. J. C. 1988 J. Chem. Phys. 88, 5051-5063.

Ladd, A. J. C. 1990 J. Chem. Phys. 93, 3484-3494.

LAM, J. 1990 J. Appl. Phys. 68, 392-403.

Mewis, J. \& MetzNer, A. B. 1974 J. Fluid Mech. 62, 593-600.

Nunan, K. C. \& Keller, J. B. 1984 J. Fluid Mech. 142, 269-287.

ONSAGER, L. 1949 Ann. N.Y. Acad. Sci. 51, 627-659.

Phillips, R. J., Brady, J. F. \& Bossis, G. 1988 Phys. Fluids 31, 3462-3472.

Shaqfeh, E. S. G. \& Fredrickson, G. H. 1990 Phys. Fluids A 2, 7-24.

Talbot, J., Kivelson, D., Allen, M. P., Evans, G. T. \& Frenkel, D. 1990 J. Chem. Phys. 92 , 3048-3057.

Zick, A. A. \& Homsy, G. M. 1982 J. Fluid Mech. 115, 13-26.

Zuzovsky, M., Adler, P. M. \& Brenner, H. 1983 Phys. Fluids 26, 1714-1723. 